\title{
Ultrafast Proton/Deuteron Dynamics in Solid Oxide Observed with Infrared Pump-Probe Spectroscopy
}

\author{
Atsunori Sakurai ${ }^{1}$, Koji Ando $^{2}$, and Satoshi Ashihara ${ }^{1, *}$ \\ ${ }^{1}$ Institute of Industrial Science, The University of Tokyo, 4-6-1 Komaba, Meguro, Tokyo 153-8505, \\ Japan \\ ${ }^{2}$ Department of Information and Sciences, Tokyo Woman's Christian University, 2-6-1 Zenpukuji, \\ Suginami, Tokyo 167-8585, Japan
}

\begin{abstract}
We performed pump-probe spectroscopy on OD stretching mode in $\mathrm{KTaO}_{3}$ to explore how a proton/deuteron interacts with the surroundings. We identified the potential anharmonicity and phonon modes which interact with a proton/deuteron, considering the temperature effect.
\end{abstract}

\section{Introduction}

Hydrogen is a ubiquitous element that plays an important role in determining the structure of molecular systems by forming hydrogen bonds. In addition, rearrangements of hydrogen bonds contribute to the structural flexibility and functionality of macromolecules like DNA or protein. Since the proton transfer involves the breaking and making of hydrogen bonds, many efforts have been made to understand the microscopic mechanism of ultrafast dynamics of hydrogen bonding and proton transfer by using infrared (IR) nonlinear spectroscopy [1].

Hydrogen is also contained in many oxide crystals as impurity [2]. Recently, a detailed microscopic understanding of proton conduction in oxides has been receiving attention from the viewpoint of applications to solid oxide fuel cells [3]. The conductivity measurement is conventionally used to understand the proton mobility, but it gives the averaged information of proton movement. Time-resolved IR spectroscopy is a promising technique to investigate ultrafast microscopic proton dynamics, because the frequency of $\mathrm{OH}$ stretching vibration is sensitive to a local hydroxyl environment. In order to explore how a proton/deuteron interacts with the surrounding environment, we employed IR pumpprobe spectroscopy for the OD stretching mode and obtained the profile of vibrational relaxation on the picosecond timescale. Additionally, we measured the temperature dependence of IR absorption spectra for OH/OD modes at various temperatures. On the basis of experimental results we discuss the vibrational relaxation mechanism, especially in terms of phonon modes which cause fluctuations on OH/OD modes.

*Corresponding author: ashihara@,iis.u-tokyo.ac.jp 


\section{Experimental Methods and Results}

\subsection{Sample}

We used the crystal of potassium tantalate $\left(\mathrm{KTaO}_{3}\right)$ as a sample. In a $\mathrm{KTaO}_{3}$ crystal, hydrogen (or deuterium) atoms make the covalent and hydrogen bonds with the first and second nearest oxygen atoms, respectively as shown in Fig. 1(a). Proton conduction in the crystal is supposed to occur by the Grotthuss mechanism, namely a series of sequential steps of $\mathrm{OH} \mathrm{O}$ transfer and $\mathrm{OH}$ orientation. $\mathrm{OH}$ and $\mathrm{OD}$ stretching modes have sharp absorption peaks at $3472 \mathrm{~cm}^{-1}$ and $2565 \mathrm{~cm}^{-1}$, respectively (Fig. 1(b)).

(a)

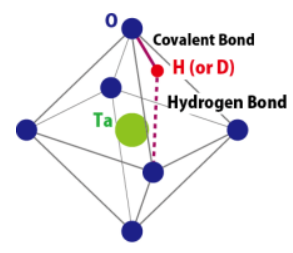

(b)

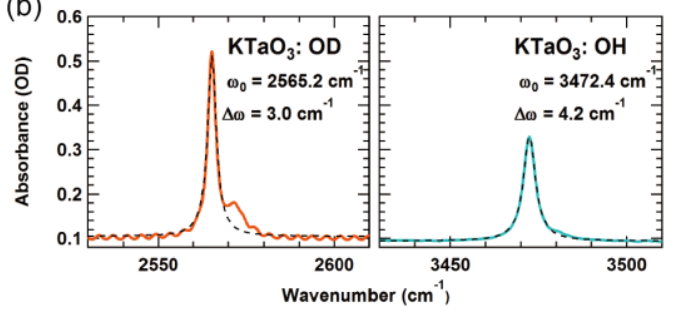

Fig. 1. (a) The crystal structure of $\mathrm{KTaO}_{3}$. Potassium atoms are not shown. (b) IR absorption spectra for OD (left) and $\mathrm{OH}$ (right) modes. Black dashed curves are the fittings with a Lorentzian function.

\subsection{IR pump-probe spectroscopy}

We performed IR pump-probe spectroscopy on OD stretching mode in $\mathrm{KTaO}_{3}$, using femtosecond mid-IR (MIR) pulses with a pulse energy of $5 \mu \mathrm{J}$.

The transient absorption change of the OD mode in $\mathrm{KTaO}_{3}$ is shown in Fig. 2(a). The bleaching signal of the fundamental transition $(v=0 \rightarrow 1)$ is observed at $2565 \mathrm{~cm}^{-1}$ due to the depletion of the ground state and the stimulated emission $(v=1 \rightarrow 0)$. Moreover, the induced absorption corresponding to the second excited state transition $(v=1 \rightarrow 2)$ is observed at $2473 \mathrm{~cm}^{-1}$. Since both signals originate from the creation of $v=1$ state, their decay times reflect its population time. Exponential fittings to them give the decay time around $200 \mathrm{ps}$ (Fig. 2(b)). This long lifetime compared with other vibrational modes in condensed phases indicates that OD mode is isolated from the phonon bands which cause the energy relaxation. We note that, although the bleaching signal of $\mathrm{OH} / \mathrm{OD}$ modes in the same material has been observed [4], this is the first observation of the 1-2 transition in solid oxide by IR pump-probe measurement. The 1-2 transition frequency is found to be redshifted by $92 \mathrm{~cm}^{-1}$, so that the potential anharmonicity is determined to be $3.6 \%$.

\subsection{Temperature dependence of IR absorption spectra}

In order to investigate the phonon mode which interacts with the OH/OD vibration, IR absorption spectra were measured for $\mathrm{OH}$ and $\mathrm{OD}$ modes at temperatures from 10 to $300 \mathrm{~K}$. The obtained spectra at different temperatures are fitted by a Lorentzian function, then the peak frequencies are evaluated. As temperature decreases, the OH/OD peak frequencies shift higher (Fig. 2(c)). The extremely narrow spectral linewidth suggests that the interaction of the $\mathrm{OH} / \mathrm{OD}$ modes with the host lattice is weak. In such a situation, a weak phonon coupling model can be applied [5]. In this model vibrational mode is weakly coupled to a single phonon band of frequency $\omega_{0}$ and spectral width $\gamma$ by a coupling 
constant $\delta \omega(\delta \omega<<\gamma)$. Under this assumption the spectral line has a Lorentzian shape which has a peak at $v(T)$ according to

$$
\tilde{\nu}(T)=\tilde{\nu}_{0}+\frac{\delta \omega}{\exp \left[\hbar \omega_{0} / k_{\mathrm{B}} T\right]-1}
$$

where $v_{0}$ is the peak position at $T=0 \mathrm{~K}$. The fittings using this equation agree well with the experimental data (Fig. 2(c)). The obtained value of $\omega_{0}$ is $150 \mathrm{~cm}^{-1}$ for both OH/OD modes and this value matches the $\Gamma_{15}$ optical phonon corresponding to a O-Ta-O bending motion [6].
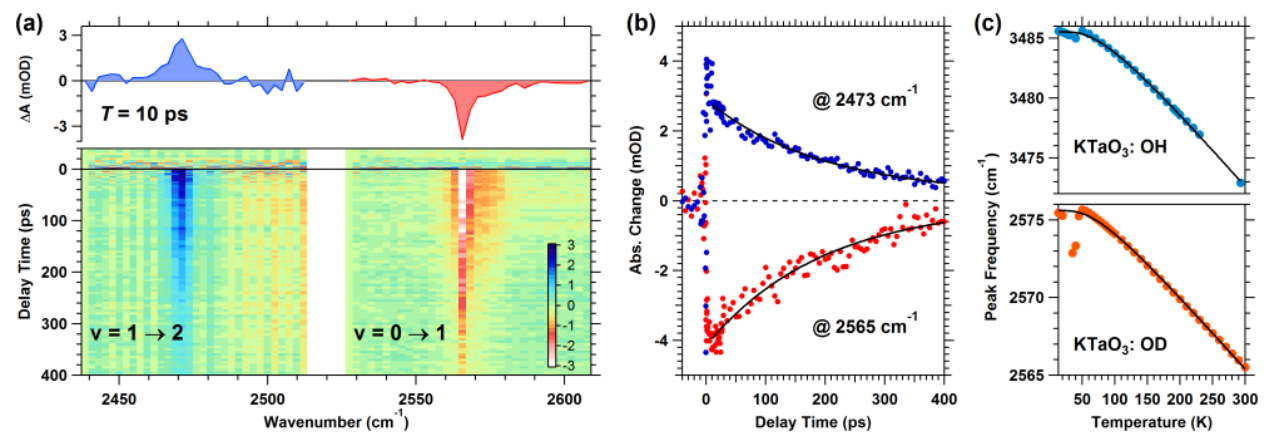

Fig. 2. (a) Two-dimensional map of the pump-probe signal of the OD mode in $\mathrm{KTaO}_{3}$. The bleaching signal of the $\mathrm{v}=0 \rightarrow 1$ transition and the induced absorption signal of the $\mathrm{v}=1 \rightarrow 2$ transition are observed. Transient absorption spectrum at $T=10 \mathrm{ps}$ is shown in the upper part. (b) Time evolution of the $0-1$ bleaching signal at $2565 \mathrm{~cm}^{-1}$ (red) and the $1-2$ excited state absorption signal at $2473 \mathrm{~cm}^{-1}$ (blue). Black curves are the exponential fittings to them. (c) Temperature dependencies of the peak frequencies of $\mathrm{OH}$ (upper) and OD (lower) modes. Black curves are the fittings with Eq. (1).

\section{Conclusion}

We observed exceptionally long population lifetime of the OD stretching mode in $\mathrm{KTaO}_{3}$. This long lifetime indicates that the OD mode is energetically isolated from lattice vibrations. From the temperature dependence of $\mathrm{OH} / \mathrm{OD}$ modes absorption, we identified the phonon mode which interacts with the proton/deuteron vibration. Furthermore, we determined the anharmonicity of potential energy surface on which proton moves. These results are valuable for understanding the microscopic mechanism of proton conduction in solid oxide.

\section{References}

1. T. Elsaesser and H. J. Bakker ed., Ultrafast Hydrogen Bonding Dynamics and Proton Transfer Processes in the Condensed Phase (Springer, Netherland, 2002).

2. M. Wöhlecke and L. Kovács, Cr. Rev. Solid State Mater. Sci. 25, 1 (2001).

3. K. D. Kreuer, Annu. Rev. Mater. Res. 33, 333 (2003).

4. E. J. Spahr, L. Wen, M. Stavola, L. A. Boatner, L. C. Feldman, N. H. Tolk, and G. Lüpke, Phys. Rev. Lett. 102, 075506 (2009).

5. B. N. J. Persson and R. Ryberg, Phys. Rev. B 32, 3586 (1985).

6. C. H. Perry, R. Currat, H. Buhay, R. M. Migoni, W. G. Stirling, and J. D. Axe, Phys. Rev. B 39, 8666 (1989). 